\title{
Impact of Covid-19 Infection on Hematological Parameters
}

\author{
Nahla A M Hamed* \\ Professor of Clinical Hematology, Faculty of Medicine, Alexandria University, Egypt
}

Submission: September 30, 2020; Published: October 29, 2020

*Corresponding author: Nahla A M Hamed, Professor of Clinical Hematology, Faculty of Medicine, Alexandria University, Egypt

\section{Abstract}

COVID-19 infection is associated with lymphopenia, occasional thrombocytopenia, and overall leukopenia at hospital admission. ICU admission and mortality risk are associated with an elevated D-dimer (dimerized plasmin fragment D) level and a decreasing lymphocyte count. Platelet count was not a discriminating test on ICU admission or during the hospitalization. Additional routine biomarkers for infected patient risk stratification and for early admission for supportive care in the ICU are urgently needed.

Abbreviations: ISTH: International Society on Thrombosis and Hemostasis; RDW: Red Blood Cell Distribution Width; ALC: Absolute Lymphocyte Count; AMC: Absolute Monocyte Count; ANC: Absolute Neutrophil Count; JAK-STAT3: Janus kinases and Signal Transducer and Activator of Transcription 3 Proteins; FDP: Fibrin Degradation Products

\section{Introduction}

Endothelial cell injury induced by viral infection activates a multitude of pro-inflammatory cytokines such as interleukin (IL)1, IL-6, and tumour necrosis factor alpha (TNF-alpha) [1]. IL-6 plays a significant role through its effect on JAK-STAT3 pathway IL-6 also enhances secretion of vascular endothelial growth factor and IL-8 but reduces E-cadherin expression on endothelial cells [2]. The proinflammatory cytokines in combination with numerous immune-active molecules including chemokines, other interleukins, colony stimulating factors and interferons contribute to a cytokine storm [2]. The cytokine storm and hyperinflammatory state results in a prothrombotic state and likely endothelial and platelet activation [3]. A subgroup of patients with COVID-19 disease may experience a fatal hypercytokinemia that often lead to multi-organ dysfunctional syndrome [1]. COVID-19 has a high rate of hospitalization and mortality [4]. Biomarkers are urgently needed for patient risk stratification [4].

\section{Hematological parameters and COVID-19}

\section{RDW and COVID-19}

Increased RDW during admission is associated with an increased mortality risk. RDW greater than $14.5 \%$ at the time of admission for SARS-CoV-2 infection was associated with an increase in mortality risk (from $11 \%$ to $31 \%$ ). The mortality riskis higher in patients ( $<70$ years) than older patients. The mechanism (s) for RDW alteration associated with COVID-19 remain unclear. Reports (on inflammation in non-COVID-19 cohorts), suggested that RDW become elevated when RBC production kinetics have slowed in the setting of increased WBC and platelet kinetics [4].

\section{Leucopenia, lymphopenia and COVID-19}

Leucopenia or normal total white cell count, lymphopenia (ALC $<1.0 \times 10^{9} / \mathrm{L}$ ) are noted in COVID-19 infected patients. Lymphopenia is believed to result from defective immune response to the virus. In addition, SARS-CoV-2 is thought to inhibit bone marrow hematopoiesis through certain receptors resulting in lymphopenia (1). Medications used for COVID-19 treatment like steroids can also cause lymphopenia [2].

\section{Thrombocytopenia and COVID-19}

Data on thrombocytopenia in COVID-19 infected patients are variable. The medium nadir platelet counts remained in the normal range in ICU and non-ICU patients [2]. The preservation of platelet count is possibly attributed to the large amounts of platelets produced in response to increased thrombopoietin formation from liver stimulation and megakaryocytes in the lung [3]. Thrombocytopenia was described in 36\% of COVID-19 hospitalised patients in one study [1]. The incidence of thrombocytopenia could be as high as $57 \%$ amongst non- 
survivors [2]. Subsequent studies have not confirmed this high frequency [3]. Mild to moderate thrombocytopenia (platelet count $>50 \times 10^{9} / \mathrm{L}$ ) are commonly seen [1]. Count below $100 \times 10$ $9 /$ l occurs in around $5 \%$ of hospitalised patients [3].

\section{Potential causes of thrombocytopenia in COVID-19 positive patients}

Thrombocytopenia is multifactorial. It could be explained by use of antibiotics, antivirals, heparin, and other commonly used agents, as well as hemodialysis and extracorporeal membrane oxygenation (ECMO). Platelet production may be affected by direct viral insult to the bone marrow and by impaired fragmentation of megakaryocytes due to COVID-19 induced damage to the lung and pulmonary capillary bed [3]. Stimulation of anti-platelet autoantibodies by SARS-Cov-2 will trigger immune-mediated platelet destruction. The antibodies and immune complexes deposited on the surfaces of platelets predispose to platelets destruction by the reticuloendothelial system [1]. In end stage COVID-19 infection multi organ failure may exacerbate thrombocytopenia [3]. Dysfunctional platelets can also contribute to increased bleeding despite being in the normal range [2]. Fibrinogen levels may fall with disease progression resulting in a hypocoagulable state and increased bleeding risk [3].

\section{Thrombosis in COVID-19 positive patients}

COVID-19 infection is associated with arterial and venous thrombosis [1]. The incidence of cerebral infarction in COVID-19 infected patients is $4.5 \%$ The incidence of DVT, PE or thrombosis elsewhere in the body has not been studied well [2]. Patients on extracorporeal membrane oxygenation and/or continuous renal replacement therapy have a higher risk of thromboembolism due to increased inflammatory process [1].

\section{Coagulation changes in COVID-19 positive patients}

There are prolongation of prothrombin time (PT) and activated partial thromboplastin time (aPTT), elevations of D dimer, fibrinogen and FDP and decreased levels of antithrombin III. aPTT prolongation is caused by increased Factor VIII level and Factor XII deficiency secondary to the presence of factor XII inhibitors. Von Willebrand factor is quantitatively increased. Lupus anticoagulant (LA) is positive in $91 \%$ of those with prolonged aPTT. The presence of both LA and Factor XII deficiency are not associated with bleeding tendency. Overt DIC (ISTH score of 5 and higher) is seen more frequently in non-survivors [1]. ADAMTS13 levels of 20-40\%, typical of other inflammatory states was found in COVID-19 without TTP [3].

\section{Mechanisms of thrombosis in COVID-19 positive patients}

The increase in inflammatory activity contributes to microvascular thrombosis including plugging of the pulmonary microvasculature and pulmonary embolism. The upregulation of tissue factor (TF) - activated VIIa complex is associated with thrombin generation and fibrin deposition in various organs including the bronchoalveolar system. Hyperactive fibrinolysis increases plasmin cleavage activity and D-dimers which correlates linearly with disease severity [1].

\section{Anti-coagulation therapy}

Prophylactic anticoagulation should be given to all hospitalised patients without evidence of active bleeding even if PT and aPTT are prolonged. The anticoagulant of choice is low molecular weight heparin, unfractionated heparin (UFH) or subcutaneous fondaprinux. UFH has little interaction with COVID-19 drugs' treatment. Its thromboprophylaxis usage is limited by the requirement for frequent aPTT monitoring. Mechanical thromboprophylaxis such as intermittent pneumatic devices should only be used if pharmacological anticoagulation is contraindicated. The use of concomitant pharmacological and mechanical anticoagulation should be avoided [1]

\section{COVID-19 infection differs from other infections}

Unlike other viral infections such as human immunodeficiency virus and cytomegalo-virus infections, the CD4/CD8 ratio was not inverted in in COVID-19 infection [5]. The aPTT may be less prolonged in COVID-19 infection as compared to typical sepsis induced disseminated intravascular coagulopathy (DIC) [1].

\section{ICU and covid 19}

Those requiring ICU care had a lower ALC and higher LDH. The median nadir of ALC is $0.4 \times 10^{9} / \mathrm{L}$, compared to $1.2 \times 10^{9} / \mathrm{L}$ in the non-ICU group [5]. Severe lymphopenia in critically ill patients is explained by lymph node destruction, suppression of lymphocytes during lactic acidosis and binding of SARS-CoV-2 to angiotensin-converting enzyme 2 receptors on lymphocytes [2]. Flow cytometry on peripheral blood lymphocytes in the ICU patients demonstrate significantly lower CD45+, CD3+, CD4+, CD8+, CD19+and CD16/56+ count [5]. The CD4/CD8 ratio was not inverted. There is activation of T-helper-1 (Th1) function due to the increased concentration of inflammatory mediators such as IL-1B, IL-6, IL-12, IL-18, IL-33, CXCL10, CCL2 and TNF-alpha [2].

During the patient stay in ICU, more profound, decrements in the level of hemoglobin, ALC and AMC occurs compared to nonICU group. The median nadir of AMC was $0.2 \times 10^{9} / \mathrm{L}$ in the ICU group, compared to $0.4 \times 10^{9} / \mathrm{L}$ in the non-ICU group. ICU patients tend to develop neutrophilia during the hospitalization (ANC of $11.6 \times 10^{9} / \mathrm{L}$, compared to $3.5 \times 10^{9} / \mathrm{L}$ in the non-ICU group). The median nadir platelet count remained in the normal range (above150 $\times 10^{9} / \mathrm{L}$ ) for both groups [5].

\section{Hematological changes during covid 19 infection and its impact on prognosis}

- $\quad$ Patients with elevated RDW at admission were 6.12 times more likely to die within 48 hours $(4.9 \%)$ than patients with a normal RDW $(0.8 \%)$. RDW may be helpful for prioritizing patients for early, aggressive intervention [4]. 
- $\quad$ Lymphopenia appears to predict COVID-19 severity [1]. An ALC approaching < $0.6 \times 10^{9} / \mathrm{L}$ may be considered as one of the indicators for early admission for supportive care in the ICU. The ICU patients had severe lymphopenia. Lymphopenia was associated with adverse outcomes and ICU stay [5].

- $\quad$ Relative lymphocytosis is often associated with a milder disease and faster onset of spontaneous recovery [1].

- $\quad$ Platelet count was not a discriminating test on ICU admission or during the hospitalization [5].

- Thrombocytopenia is a marker of poor clinical outcome and was associated with a three-fold enhanced risk of worsening disease. the platelet count was lower in patients with very severe COVID-19. Twenty percent of COVID-19 patients who died had a platelet count $<100 \times 10 \% /$, compared with $1 \%$ of survivors. Very low platelet counts of $<20 \times 10^{9} / \mathrm{l}$, or a sudden fall in the platelet count $>50 \%$ over $24-48$ hours can occur in the pre-terminal stages of COVID-19 [3].

- Increasing values in WBC, ALC, AMC, and down trending LDH was noted in ICU as clinical condition improve [5].

- Coagulopathic features are associated with a more severe outcome in COVID-19 infection [1]. A high D-dimer value correlates with ICU requirement and higher mortality compared to individuals with normal/mild elevation of the D-dimer levels [2] A fourfold increase in D-dimer predicts mortality in COVID-19 infection due to the increased risk of venous thrombosis and cytokine storm [5].

- $\quad$ Serum ferritin levels were significantly associated with disease severity and outcome [2].

- Other biochemical markers associated with a more severe presentation are elevations in lactate dehydrogenase $(\mathrm{LDH}), \mathrm{C}$ reactive protein, procalcitonin, Troponin $\mathrm{T}$, creatinine, and liver enzymes [5].

\section{Challenges in Diagnosis}

- $\quad$ Almost $30 \%$ of adults and many children infected by COVID-19 are asymptomatic [1]. A proportion of asymptomatic patients with positive RT-PCR results are admitted during contact tracing. They may not have significant $\mathrm{CBC}$ anomalies at presentation. This may result in lower hematological indices on admission compared to patients in critical condition [5].

- Further studies should be conducted correlating the clinical condition to laboratory findings [5] in patients with low viral load during the early phase of the disease (Day 1-4) and also in patients who are recovering from the infection but still infectious [1].

- $\quad$ Recognizing how many patients had hemato-oncological disorders at baseline like immune thrombocytopenia, or any cancer receiving chemotherapy affecting the bone marrow hematopoiesis is essential [2].

\section{Conclusion}

It is recommended that every patient diagnosed with COVID-19 infection should have a baseline PT/aPTT, D dimer and platelet count. Monitoring of hematologic parameters may help to identify patients who may need ICU care.

\section{References}

1. Kasinathan G, Sathar J (2020) Hematological manifestations, mechanisms of thrombosis and anticoagulation in COVID-19 disease: A review. Annals of medicine and surgery 56: 173-177.

2. Sahu KK, Siddiqui AD (2020) From Hematologist's desk: The effect of COVID-19 on blood system. Am J Hematol 95(8): E213-E215.

3. Pavord S, Cooper N, Thachil J, Hunt B, Murphy M, et al. (2020) Practical guidance for the management of adults with immune thrombocytopenia during the COVID-19 pandemic. Br J Haematol 189(6): 1038-1043.

4. Foy BH, Carlson JCT, Reinertsen E, Valls RPI, Lopez RP, et al. (2020) Association of red blood cell distribution width with mortality risk in hospitalized adults with SARS-CoV-2 infection. JAMA Network Open 3(9): e2022058.

5. Fan BE, Chong VCL, Chan SSW, Lim GH, Lim KGE, et al. (2020) Hematologic parameters in patients with COVID-19 infection. Am J Hematol 95(6): E131-E134.

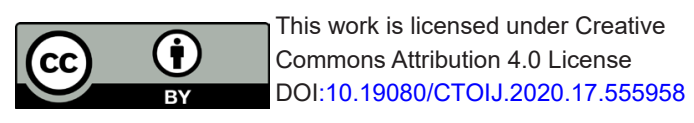

\begin{tabular}{l} 
Your next submission with Juniper Publishers \\
will reach you the below assets \\
- Quality Editorial service \\
- Swift Peer Review \\
- Reprints availability \\
- E-prints Service \\
- Manuscript Podcast for convenient understanding \\
- Global attainment for your research \\
- Manuscript accessibility in different formats \\
( Pdf, E-pub, Full Text, Audio) \\
- Unceasing customer service \\
Track the below URL for one-step submission \\
https://juniperpublishers.com/online-submission.php \\
\hline
\end{tabular}

